\title{
Helper-dependent adenovirus-mediated gene transfer of a secreted LDL receptor/transferrin chimeric protein reduces aortic atherosclerosis in LDL receptor-deficient mice
}

\author{
Eleonora Leggiero $^{1} \cdot$ Giuseppe Labruna $^{2} \cdot$ Laura laffaldano $^{1,3} \cdot$ Barbara Lombardo $^{1,3} \cdot$ Adelaide Greco $^{1,4,5}$. \\ Dario Fiorenza $^{2}$ - Matteo Gramanzini ${ }^{1,5} \cdot$ Donatella Montanaro $^{1}$ - Alfonso Baldi ${ }^{1,6}$ - Vincenzo Cerullo (iD ${ }^{1,3,7}$. \\ Lucia Sacchetti $^{1} \cdot$ Lucio Pastore $^{1,3}$
}

Received: 3 July 2018 / Revised: 2 November 2018 / Accepted: 23 November 2018 / Published online: 30 January 2019

๑) Springer Nature Limited 2019

\begin{abstract}
Familial hypercholesterolemia (FH) is a genetic hyperlipidemia characterized by elevated concentrations of plasma LDL cholesterol. Statins are not always effective for the treatment of FH patients; unresponsive patients have poor prognosis and rely on LDL apheresis. In the past, we developed safe and effective gene therapy strategies for the expression of antiatherogenic proteins using PEGylated helper-dependent adenoviral (HD-Ad) vectors. We recently developed a HD-Ad vector for the expression of the soluble form of the extracellular portion of the human LDL receptor (LDLR) fused with a rabbit transferrin dimer (LDLR-TF). We evaluated the efficacy of the LDLR-TF chimeric protein in CHOLDLA7, a cell line lacking LDLR expression, restoring the ability to uptake LDL. Subsequently, we administered intravenously $1 \times 10 \mathrm{E} 13 \mathrm{vp} /$ $\mathrm{kg}$ of this vector in LDLR-deficient mice and observed amelioration of lipid profile and reduction of aortic atherosclerosis. Finally, we studied LDL distribution after HD-Ad vector-mediated expression of LDLR-TF in LDLR-deficient mice and found LDL accumulation in liver, and in heart and intestine. These results support the possibility of lowering LDL-C levels and reducing aortic atherosclerosis using a secreted therapeutic transgene; the present strategy potentially can be modified and adapted to non-systemic gene transfer with expression of the secreted chimeric protein in muscle or other tissues. Intramuscular or local administration strategies could improve the safety profile of this strategy and facilitate applicability.
\end{abstract}

Supplementary information The online version of this article (https:// doi.org/10.1038/s41434-019-0061-z) contains supplementary material, which is available to authorized users.

Lucio Pastore

lucio.pastore@unina.it

1 CEINGE-Biotecnologie Avanzate, Napoli, Italy

2 IRCCS SDN, Napoli, Italy

3 Dipartimento di Medicina Molecolare e Biotecnologie Mediche, Università degli Studi di Napoli Federico II, Napoli, Italy

4 Dipartimento di Scienze Biomediche Avanzate, Università degli Studi di Napoli Federico II, Napoli, Italy

5 Istituto di Biostrutture e Bioimmagini, CNR, Napoli, Italy

6 Department of Environmental, Biological and Pharmaceutical Sciences and Technologies, University of Campania "Luigi Vanvitelli”, Caserta, Italy

7 Laboratory of ImmunoViroTherapy, Centre for Drug Research (CDR), Division of Pharmaceutical Biosciences, Faculty of Pharmacy, University of Helsinki, Helsinki, Finland

\section{Introduction}

Coronary artery disease is the leading cause of death in western countries. One of the major predisposing factors is the increase of pro-atherogenic lipoproteins. The most characterized genetic hyperlipidemia is familial hypercholesterolemia $(\mathrm{FH})[1,2]$ mainly due to mutations in the lowdensity lipoprotein (LDL) receptor $(L D L R)$ gene with consequent increase in LDL cholesterol (LDL-C) and lipid accumulation in tendons (xanthomas) and arteries (atheromas) [3]; mutations in other genes such as $A P O B$ and proprotein convertase subtilisin/kexin type 9 (PCSK9), also give rise to a similar phenotype $[4,5]$.

Treatment for heterozygotes and homozygotes is directed at lowering the plasma level of LDL. In heterozygotes, the most effective therapy is the administration of drugs called statins, whereas homozygotes are relatively resistant to drugs and their plasma LDL levels can be effectively lowered by physical or surgical means. Treatment in heterozygotes and homozygotes can lead to a reduced rate of 
progression, and, regression of coronary atherosclerosis but is not effective in all patients, justifying the search for more effective alternatives. New drugs for FH have recently been approved: mipomersen, an antisense oligonucleotide inhibitor of apo B-100 mRNA, administered subcutaneously once weekly, has also been approved for use in the Homozygous familial hypercholesterolaemia $(\mathrm{HoFH})$ population from the Food and Drug Administration [6]; lomitapide is a microsomal triglyceride transfer protein (MTP) inhibitor, binding directly to MTP in the lumen of the endoplasmic reticulum, developed as an orphan drug for treatment of $\mathrm{HoFH}$, and is indicated in addition to low-fat diet and other lipid-lowering agents, with or without LDL apheresis, as an adjunctive treatment for the management of $\mathrm{HoFH}$ in the United States and European Union [7]. Currently available PCSK9 inhibitors, human monoclonal antibodies that exert their effect by binding to PCSK9, blocking the lysosomal degradation of LDLRs, and allowing for their repeated intracellular recycling, has been recently introduced and are able to reduce LDL-C in patients with heterozygous or receptor-defective homozygous $\mathrm{FH}$ by reducing LDLR degradation [8]. In contrast with the abovementioned therapeutic strategies, gene therapy can be considered as a definitive cure and not a life-long patient care, particularly for homozygous $\mathrm{FH}$ patients, aiming possibly at longlasting effects that lead to a definitive therapeutic solution. Significant progresses in gene transfer technology have encouraged to develop LDLR gene therapy approaches for FH treatment [9-12]. In experimental animal models of FH, VLDL receptor overexpression following viral vector-based gene transfer is associated with long-term stable correction of hyperlipidemia, with consequent attenuation of atherosclerosis progression and in certain cases even with lesions regression $[13,14]$. We also observed that helper-dependent adenoviral (HD-Ad)-mediated human apolipoprotein A-I (hApoA-I) overexpression reduces aortic atherosclerosis development in ApoE- and LDLR-deficient mice [15, 16].

More recently, an AAV8-derived vector expressing LDLR has been successfully evaluated in the mouse and rhesus monkey FH models [17]. However, albeit at a much lower levels compared to Ad vectors, high concentrations of AAV vectors induce a certain degree of systemic toxicity [18]. On the other hand, both in animal models and in humans, intramuscular (IM) delivery of viral vectors has always resulted in mild local inflammation and toxicity [19].

We therefore decided to evaluate the efficacy of an alternative therapeutic strategy for $\mathrm{FH}$ treatment based on a chimeric secreted protein expressed after HD-Ad vector cell transduction; the advantage of this approach compared to LDLR gene transfer consists in the theoretical possibility of the expression of the therapeutic transgene in alternative tissues, such as muscle, to reduce treatment toxicity and to translate this approach into human application. The secreted chimeric protein specifically binds LDL and is internalized by several mammalian cells, including hepatocytes, with a receptor-mediated endocytosis mechanism functionally similar to that involved in LDL metabolism. Razzini et al., in fact, generated this fusion protein that contains the signal peptide, the LDL-binding domain, the epidermal growth factor (EGF) homology domain, and the O-linked sugar domain of the human LDLR, lacking the membrane anchor as well as the cytoplasmic domain; the LDLR portion is fused with a dimer of rabbit transferrin [20, 21]. Transferrin receptor is also known to be recycled, therefore a mechanism similar to what observed with LDLR should occur [22].

Using a non-viral approach, they demonstrated that this fusion protein binds LDL restoring uptake in LDLRdeficient Watanabe heritable hyperlipidemic rabbit fibroblast in vitro [20]. The chimeric protein induces endocytosis of LDL bound to the extracellular LDLR portion through the interaction of the transferrin dimer portion with the transferrin receptors (TfRs) [20]. In vivo hydrodynamic administration of a plasmid for the expression of the LDLRTF fusion protein was able to induce liver uptake of labeled LDL in LDLR-deficient mice [20]. In this case, plasmid administration induced only a transient effect in LDLRdeficient mice. In fact, hydrodynamic administration of unmodified plasmids allows low levels of hepatocyte transduction with short duration of expression; therefore, modifications of LDL-C levels and aortic atherosclerosis were not observed with this approach [20]. It was nonetheless relevant that most of the labeled LDL were uptaken by the liver, with limited lipid accumulation in other sites [20].

We have developed a HD-Ad vector for the expression of the above-mentioned chimeric LDLR-TF protein to achieve safe and long-term transgene expression; in the present work, we evaluated the effect of HD-Ad-mediated expression of LDLR-TF in LDLR-deficient mice. We observed amelioration of lipid profiles and reduction of aortic atherosclerosis in treated mice supporting possible developments of this strategy for future clinical applications.

\section{Materials and methods}

\section{Development of a HD-Ad vector for LDLR-TF expression}

We generated a cassette for liver-specific expression of the LDLR-TF fusion protein. At this aim, we used the mouse transthyretin (TTR) promoter to drive LDLR-TF expression. The expression cassette was introduced into the 
pLPBL1 plasmid and further subcloned into the $\mathrm{p} \Delta 28$ backbone plasmid to generate the HD-AdTTR-LDLR-TF as described [15]. Rescue and amplification of the vector were performed using the Ad-NG163R-2 helper virus as described elsewhere [23-25]. Briefly, a 60-mm dish of 116 cells at $80 \%$ confluence was transfected with Lipofectamine 2000 with $5 \mu \mathrm{g}$ of PmeI-digested parental plasmid. Next day, cells were infected with Ad-NG163R-2 at a multiplicity of infection (MOI) of $1,000 \mathrm{vp} / \mathrm{cell}$. The vector was amplified by serial coinfections of $60-\mathrm{mm}$ dishes of 116 cells at $90 \%$ confluence with $10 \%$ of the crude lysate from the previous passage and Ad-NG163R-2 at an MOI of $200 \mathrm{vp} /$ cell. After three passages (P) in 60-mm dishes (P1, P2, and P3), one 150-mm dish of 116 cells at $90 \%$ confluence was co-infected with $10 \%$ of the crude serial P3 lysate and Ad-NG163R-2 at MOI of $200 \mathrm{vp} /$ cell for P4. Large-scale HD-Ad production was performed in a 3 liter-culture of 116 cells $(3-4 \times 10$ E5 cells $/ \mathrm{ml})$ in spinner flask co-infected with all crude lysate from the 150-mm dish of serial P4 and Ad-NG163R-2 at a 200 vp/cell MOI; coinfected cells were harvested and resuspended in TM solution (10 mM Tris- $\mathrm{HCl}$ and $2 \mathrm{mM} \mathrm{MgCl}_{2}, \mathrm{pH}$ 8.0) 48 hours after infection. The harvested cells were lysed with three freezethaw cycles and incubated in $2 \mathrm{M} \mathrm{MgCl}_{2}$ and DNase I for $1 \mathrm{~h}$ at $37^{\circ} \mathrm{C}$. Subsequently, cellular debris were spun down and lysate was subjected to ultracentrifugation, as described elsewhere [14]. Vector concentration was measured as virus particle number determining absorbance at $260 \mathrm{~nm}$. Helper virus contamination and vector characterization were performed as previously described [26].

\section{Cell cultures and transfections}

The 116 cells (provided by Dr. Ng from Baylor College of Medicine, Houston, Texas) were cultured in minimum essential medium containing $10 \%$ fetal bovine serum, $50 \mathrm{U}$ penicillin $/ 50 \mu \mathrm{g}$ streptomycin in a humidified incubator at $37^{\circ} \mathrm{C}$ with $5 \% \mathrm{CO}_{2}$. Cells were seeded in $60-\mathrm{mm}$ dishes and transfected the following day with Lipofectamine 2000 transfection reagent (Life Technologies, USA), according to the manufacturer's instructions.

\section{Fluorescent LDL incorporation assay}

Fluorescently labeled LDL (DiI-LDL, Molecular Probe by Life Technologies, USA) are added to CHOldlA7 cells (gift from Dr. Krieger) [27], a cell line deficient for the LDLR, at a $10 \mu \mathrm{g} / \mathrm{ml}$ concentration for 5 hours, after infection with either HD-AdTTR-LDLR-TF vector $(10 \mathrm{vp} / \mathrm{cell})$ or with mock vector $(10 \mathrm{vp} / \mathrm{cell})$. Complementation of CHOldlA7 cell defect was evaluated by assessing incorporation of fluorescently labeled LDL (Dil-LDL) with intracellular fluorescence with a confocal microscopy, LSM (Laser Scanning Microscope) 510 Meta (Zeiss).

\section{Immunofluorescence staining}

293 cells were washed with phosphate-buffered saline (PBS), fixed using 4\% paraformaldehyde for $15 \mathrm{~min}$ at room temperature, and washed again with PBS. Cells were permeabilized using $0.2 \%$ Triton $\mathrm{X}-100$ in PBS for $15 \mathrm{~min}$ at room temperature, followed by washing the samples with PBS. Samples were incubated for 1 hour at room temperature in blocking buffer (PBS with $10 \%$ bovine serum albumin). Anti-transferrin antibody (Abcam ab9033, England) was used as primary antibody and incubated in blocking for 2 hours at room temperature; after washing with PBS cells were incubated for $45 \mathrm{~min}$ at room temperature with an anti-rabbit secondary antibody diluted in blocking buffer [21].

\section{Western blot analysis}

Two microliters of plasma were combined with Laemmli buffer and electrophoresed on a 6.5\% PAGE. Samples were then transferred to nitrocellulose filters and subjected to western blotting analysis. We used a primary anti-rabbit transferrin ab (Abcam ab9033, England) diluted 1:500 in blocking buffer; as secondary antibody, we used a horseradish peroxidase (HRP)-conjugated rabbit anti-goat IgGs (Abcam ab97100, England) diluted $1: 10,000$ in blocking buffer. Antigen-antibody complexes were revealed with an enhanced chemiluminescent substrates for detection of HRP enzyme activity (Thermo Scientific Pierce), according to the manufacturer's instructions [21].

\section{Animal studies}

Animal studies were performed in accordance with National Institutes of Health guidelines and in accordance with ethical and safety rules and guidelines for the use in biomedical research provided by the relevant Italian laws and European Union's directives (no.86/609/EC). All efforts were made to minimize the animal's suffering.

Food and water were provided ad libitum. For all the experiments, we used 8-week-old female LDLR-deficient mice in C57BL/6 background (Jackson Laboratory). Animals were placed under an infrared lamp for $6 \mathrm{~min}$, in order to induce tail vein vasodilation. A unit of $1 \times 10 \mathrm{E} 13$ $\mathrm{vp} / \mathrm{kg}$ of HD-Ad vector was diluted in sterile PBS, prewarmed at $37^{\circ} \mathrm{C}$, and injected into tail vein as previously described [28]. Injections were performed in a total volume of $200 \mu \mathrm{l}$. Control mice were treated with the same volume of PBS. Blood samples $(200 \mu \mathrm{l})$ were withdrawn at $0,1,2,4,8$, and 12 weeks from the retro-orbital plexus, collected in a $1.5 \mathrm{ml}$ tube containing $20 \mu \mathrm{l}$ of $50 \mathrm{mM}$ EDTA ( $\mathrm{pH}$ 8.0) and centrifuged at low speed to obtain 
plasma. Plasma lipid profile (tryglicerides: TG; total cholesterol: TC; HDL-cholesterol: HDL-C; and LDL-C) was determined by dry chemistry technology on a Vitros 250 Analyzer (Ortho Clinical Diagnostics, Johnson \& Johnson Co, Rochester, NY). For quality control, we used two commercial control sera (Ortho Clinical Diagnostics) to detect potential inaccuracy of the methods on both low and high analytes concentrations (low concentration control intra assay standard deviation range for the tested analytes: 1.56-4.33; high concentration control intra assay standard deviation range: 2.35-6.30; data from control assay sheets). During Fluorescence molecular tomography (FMT) studies, animals were maintained in general anesthesia at $37^{\circ} \mathrm{C}$ body temperature. Anesthesia was initially delivered in an induction chamber saturated with $5 \%$ isoflurane (Iso-Vet $1000 \mathrm{mg} / \mathrm{g}$ Inhalation Vapor, Piramal Healthcare UK Ltd., Northumberland, UK) in oxygen ( $2 \mathrm{l} / \mathrm{min}$ ) and subsequently maintained during all procedures with $2.5 \%$ isoflurane in oxygen $(2 \mathrm{l} / \mathrm{min})$.

\section{Quantification of aortic atherosclerotic lesions}

After sacrifice, aortas were dissected from heart to iliac branching, with particular attention at removing external fat in order to stain exclusively sub-intimal aortic fat [14]. Aortic lesion stainings were performed as previously described [29]; images were acquired using a Nikon Coolscope (Nikon), and fat deposits were quantified with NIS elements software (Nikon). For histological analysis, aortas were formalin-fixed and paraffin-embedded. Representative sections of each specimen were stained with hematoxylineosin and were examined by a pathologist to evaluate atherosclerotic lesions.

\section{Preparation of VivoTag 750S-labeled LDL nanoparticle}

Lyophilized LDL (3,500 kDa, Sigma Aldrich) were dissolved overnight in $50 \mathrm{mM}$ carbonate/bicarbonate buffer, $\mathrm{pH} 8.5$, at a concentration of $1 \mathrm{mg} / \mathrm{ml}$, with gentle rotation. One milligram of VivoTag ${ }^{\oplus} 750 \mathrm{~S}$ (PerkinElmer), a near-infrared (NIR) fluorescent imaging labeling agent containing an $N$-hydroxysuccinimide-ester, was reconstituted with $100 \mu$ of dimethyl sulfoxide in a dark vial. One milliliter of LDL solution was incubated with $10 \mu \mathrm{l}$ of VivoTag ${ }^{\circledR} 750 \mathrm{~S}$ solution for 1 hour without light exposure at room temperature. After incubation, the reaction mix was purified with a size exclusion chromatography using Sephadex ${ }^{\mathrm{TM}}$ G-25 Medium PD-10 Desalting (GE Healthcare). Elution was monitored measuring protein concentration spectrophotometrically at 280 and 750 nm. After purification, we obtained a LDL concentration of $0.5 \mathrm{mg} / \mathrm{ml}$ with $8.5 \mu \mathrm{M}$ of fluorophore. The solution was stored at $4{ }^{\circ} \mathrm{C}$ in a dark vial.
Fluorescence in vivo studies with in VivoTag 750Slabeled LDL nanoparticles

LDL biodistribution in vivo was evaluated using the FMT $4000^{\mathrm{TM}}$ fluorescence tomography in vivo imaging system (PerkinElmer, Massachusetts, USA). For whole-body imaging, anesthetized mice were placed in a prone position on the imaging cassette, adjusted to the proper depth to gently restrain each mouse, and then inserted into the heated docking system (regulated at $\sim 37^{\circ} \mathrm{C}$ ). A NIR laser diode was used to transilluminate mouse bodies, with signal detection occurring via a thermoelectrically cooled chargecoupled device camera placed on the opposite side of the animal. Scanning was performed using the 750EX channel. The entire image acquisition sequence took approximately 6-8 min per mouse. The images were reconstructed using the TrueQuant software for qualitative and quantitative three-dimensional analysis of the total amount of fluorescence signal in each mouse (pmoles). Mice blood clearance, organ distribution, and in vivo imaging were investigated.

\section{Statistics}

Results were analyzed using the GraphPad Prism 5 Software (Graphpad Software); $p<0.05$ was considered statistically significant. Kolmogorov-Smirnov test was used to estimate normal data distribution. Intra-group differences between 0-, 1-, 2-, 4-, 8-, and 12-week time points were evaluated by analysis of variance analysis followed by Bonferroni correction. Inter-group differences between treated and untreated mice were evaluated by Student's $t$ test. The equality of variances was estimated by the Levene's test. All data are expressed as mean \pm SD.

\section{Results}

\section{HD-Ad vector-mediated LDLR-TF expression induces LDL uptake in a cellular FH model}

We generated a cassette for a liver-specific expression of the LDLR-TF fusion protein under the control of the mouse TTR promoter to reduce host response to the transgene. We then developed a HD-Ad vector containing this cassette for subsequent in vitro and in vivo evaluation (HD-AdTTRLDLR-TF). We confirmed expression of the fusion protein, infecting 293 cells with either the HD-AdTTR-LDLR-TF vector $(10 \mathrm{vp} /$ cell $)$ or a mock vector $(10 \mathrm{vp} /$ cell $)$ as negative control and analyzed cells by immunofluorescence 48 hours after infection with an anti-TF antibody. Cells infected with HD-AdTTR-LDLR-TF showed immunoreactivity in perinuclear structures, likely the endoplasmic reticulum, as expected for a protein channeled through the secretory 


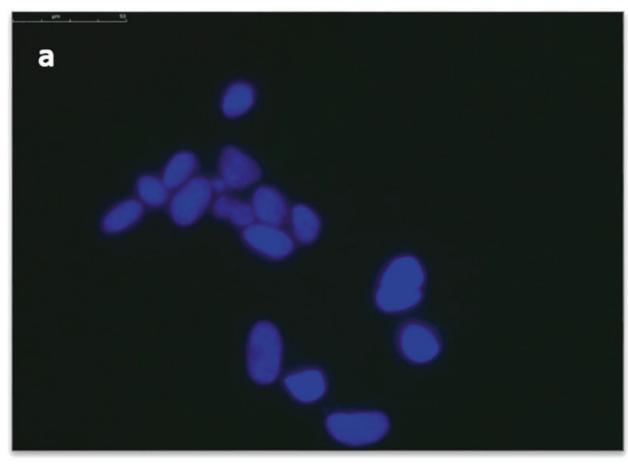

Fig. 1 In vitro transduction with Hd-AdTTR-LDLR-TF allows expression of the chimeric protein. 293 cells were infected with a control vector (a) or with HD-AdTTR-LDLR-TF (b); immunofluorescence staining was performed with an antibody directed to

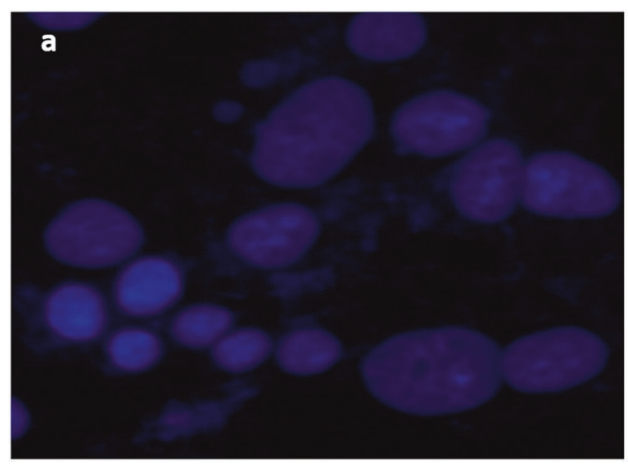

Fig. 2 Infection with HD-AdTTR-LDLR-TF restores low-density lipoprotein (LDL) uptake in CHOldlA7 cells. CHOldlA7 cells (a cell line deficient for the LDL receptor (LDLR)) were infected with HDAdTTR-LDLR-TF or with a control vector and supernatants were collected; LDL uptake was determined assessing the incorporation of fluorescently labeled LDL (DiI-LDL) in CHOldlA7 cells treated with

pathway (Fig. 1). This result indeed suggests that a significant portion of fusion proteins may be contained in endocytic vesicles. We then evaluated the ability of the LDLR-TF fusion protein to restore LDL uptake in the LDLR-deficient CHOldlA7 cell line. At this purpose, we evaluated the incorporation of fluorescently labeled LDL (Dil-LDL) in CHOldlA7 cells, treated with the supernatant of cells infected with either HD-AdTTR-LDLR-TF or a control vector: as expected, CHOldlA7 cells treated with the supernatant containing the fusion protein recover the ability to uptake LDL (Fig. 2).

\section{HD-Ad vector-mediated LDLR-TF expression ameliorates lipid profile and reduces aortic atherosclerosis in LDLR-deficient mice}

After assessing the ability of the LDLR-TF transgene in complementing LDLR function in vitro, we decided to evaluate its efficacy in vivo. At this aim, we intravenously

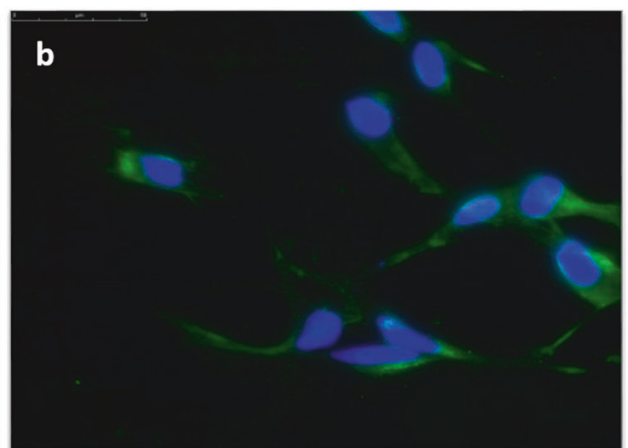

rabbit transferrin: association with perinuclear structures in cells infected with HD-AdTTR-LDLR-TF suggests that significant portion of fusion protein is contained in endocytotic vesicles localized to the perinuclear region

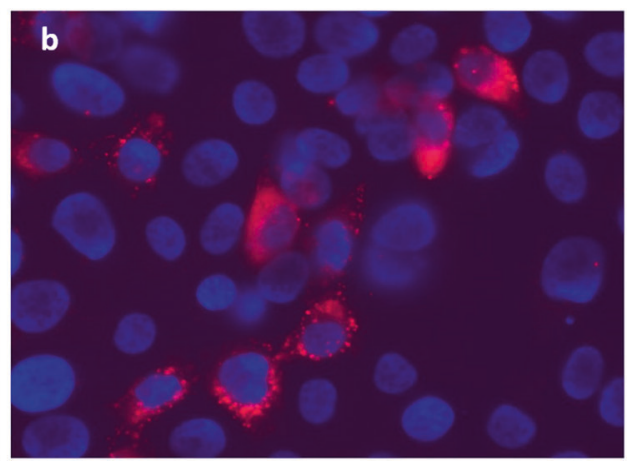

supernatant obtained from cells infected with either a control vector (a) or HD-AdTTR-LDLR-TF vector (b). DiI-LDL-positive endocytic vesicles were observed in cells treated with the LDLR-TF-containing supernatant; the incorporation of DiI-LDL was analyzed by confocal microscopy

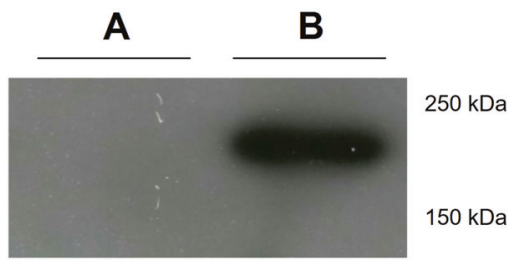

Fig. 3 HD-AdTTR-LDLR-TF administration induced LDLR-TF expression in low-density lipoprotein receptor (LDLR)-deficient mice. Two groups of LDLR-deficient mice were injected with either $1 \times 10 \mathrm{E} 13 \mathrm{vp} / \mathrm{kg}$ of HD-AdTTR-LDLR-TF vector or phosphatebuffered saline as control; LDLR-TF fusion protein was detected in blood sample from treated mice by western blot analysis using an antirabbit transferrin antibody. A band of approximately $200 \mathrm{kD}$ was detected in the plasma of mice treated with the HD-AdTTR-LDLR-TF vector (b) that was absent in plasma from control mice (a)

administered $1 \times 10 \mathrm{E} 13 \mathrm{vp} / \mathrm{kg}$ of HD-AdTTR-LDLR-TF to a group of LDLR-deficient mice; a control group was treated with PBS. We collected blood samples from both 

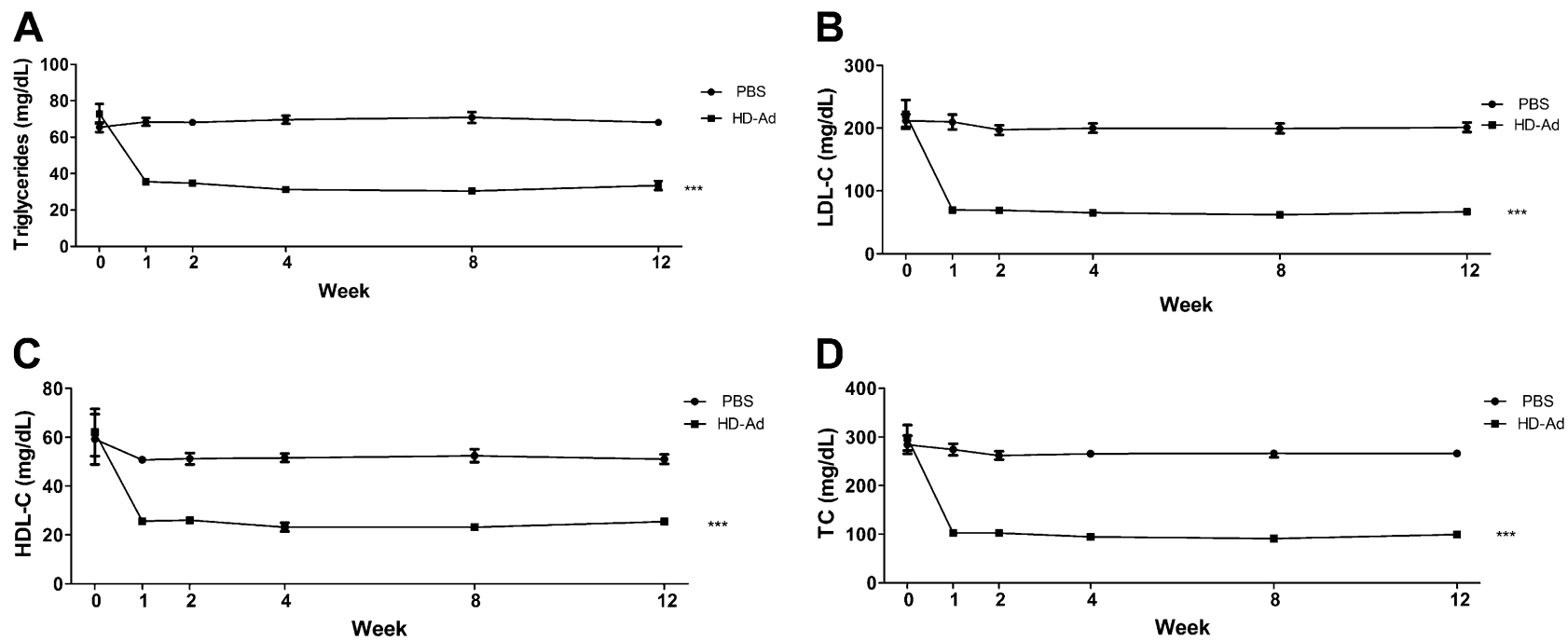

Fig. 4 HD-Ad-mediated LDLR-TF expression ameliorate lipid profiles in low-density lipoprotein receptor (LDLR)-deficient mice. A group of LDLR-deficient mice $(n=5)$ was treated intravenously with $1 \times 10$ E13 $\mathrm{vp} / \mathrm{kg}$ of HD-Ad/TTR-LDLR-TF (solid square), whereas a second group of mice $(n=5)$ was treated with the same volume of phosphatebuffered saline (PBS) as control (solid circle). Samples were collected

before treatment and 1, 2, 4, 8, and 12 weeks after treatment. Triglyceride (a), total cholesterol (TC) (b), high-density lipoprotein cholesterol (HDL-C) (c), and low-density lipoprotein cholesterol (LDL-C) (d) levels were determined in both groups. TC, HDL-C, and LDL-C levels were significantly different between treated mice and the control group (*** $p<0.0001)$. Data are expressed as mean \pm SD

groups at different time points. We assessed the presence of the fusion protein in plasma from mice treated with HDAdTTR-LDLR-TF by western blot using a commercially available polyclonal antibody against TF. We detected a prominent band of approximately $200 \mathrm{kD}$ absent in mocktreated mice (Fig. 3); we observed an increase in molecular weight, probably due to glycosylation as previously reported [17]. To evaluate the effect of LDLR-TF expression on lipid metabolism, we determined TG, TC, LDL-C, and HDL-C levels at different time points $(0,1,2$, 4,8 , and 12 weeks) in both groups of mice. Baseline levels of plasma TG were $72.8 \pm 5.4$ and $65.4 \pm 2.6 \mathrm{mg} / \mathrm{dl}$ in HDAdTTR-LDLR-TF-treated animals and in the control group, respectively; we observed a statistically significant reduction of the levels of $\mathrm{TG}$ in vector-treated mice compared to controls (Fig. 4a). Basal LDL-C levels were similar in both groups $(176.7 \mathrm{mg} / \mathrm{dl}$ in HD-Ad-treated mice and $211.2 \mathrm{mg} / \mathrm{dl}$ in the control group); in treated mice, LDL-C levels started to decrease 1 week after vector administration and remained significantly lower for the entire duration of the experiment (Fig. 4b). Basal levels of HDL-C were also similar in the two groups $(62 \pm 9.7 \mathrm{mg} / \mathrm{dl}$ in HD-Ad-treated mice and $59.2 \pm 10.3 \mathrm{mg} / \mathrm{dl}$ in the control group); however, 1 week after treatment also, HDL-C values decreased in vector-treated mice and remained lower for the entire duration of the experiment (Fig. 4c); this reduction is expected since LDLR-deficient mice have also increased LDL compared to wild-type mice. Basal levels of TC were $298.4 \pm 26.2 \mathrm{mg} / \mathrm{dl}$ in vector-treated

mice and $284 \pm 18.5 \mathrm{mg} / \mathrm{dl}$ in the control group; 1 week after administration HD-Ad-treated mice showed a significant decrease in TC levels to $102 \pm 4.3 \mathrm{mg} / \mathrm{dl}$; the control group of mice did not show any significant variation of TC levels (Fig. 4d). These data suggest that HDAd-mediated LDLR-TF expression induces a long-lasting amelioration of lipid profile in LDLR-deficient mice. We also verified whether the treatment had an effect on the iron status of the mice, but we observed no significant differences of free iron concentration before and after treatment (data not shown).

Lastly, we evaluated whether changes in lipid metabolism affected development of atherosclerotic lesions. Twelve weeks after treatment we sacrificed both group of mice and dissected aortas from heart to iliac branching. At a macroscopic evaluation, we observed deposits of fat at the level of three branches of the aorta as expected (brachiocephalic trunk, left common carotid artery, and left subclavian artery). Fat deposits were much more evident in control mice compared to vector-treated mice (Fig. 5a). To quantify atherosclerotic lesions, we stained the aortas enface with Oil-Red-O after removal of external fat and residual tissues [14]. Lesion areas were significantly smaller in HD-AdTTR-LDLR-TF vector-treated with $(1.78 \pm 0.48$ $\left.\mathrm{mm}^{2}\right)$ compared to control group $\left(5.38 \pm 0.54 \mathrm{~mm}^{2}\right.$, Fig. 5b). Histological analysis of aortas, by means of hematoxylin/eosin staining confirmed the reduction of atherosclerotic lesions in animals treated with HD-Ad/TTRLDLR-TF (Supplemental Fig. 1, panel A), respect to the 

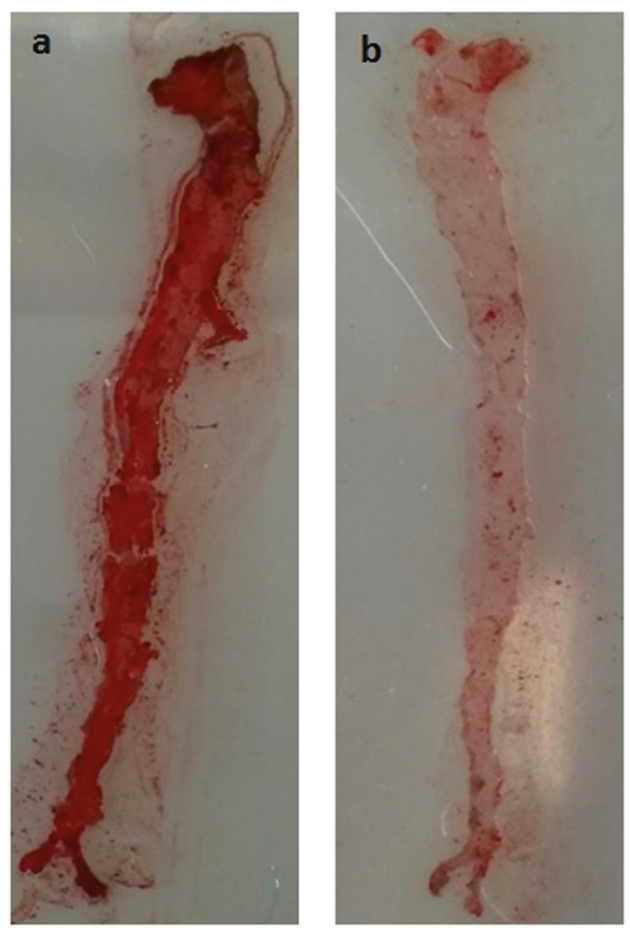

Fig. 5 HD-Ad-mediated LDLR-TF expression reduces aortic atherosclerosis development. A group of LDLR-deficient mice $(n=5)$ were treated intravenously with $1 \times 10 \mathrm{E} 13 \mathrm{vp} / \mathrm{kg}$ of HD-Ad/TTR-LDLRTF-treated mice, whereas a second group of mice $(n=5)$ was treated with the same volume of phosphate-buffered saline (PBS) as control. Twelve weeks after treatment, mice were sacrificed and their aortas were dissected. Sub-intimal fat deposits in the aortas were determined after removal of external fat and staining with Oil-Red-O. After gross

control group (Supplemental Fig. 1, panel B). These results strongly suggest that Hd-AdTTR-LDLR-TF administration leads not only to persistent transgene expression and amelioration of lipid metabolism, but also to a significant reduction in aortic atherosclerosis formation.

\section{LDL biodistribution after Hd-AdTTR-LDLR-TF vector administration in LDLR-deficient mice}

Razzini et al. previously showed that LDLR-TF expression after hydrodynamic injection of an expression plasmid induced LDL accumulation in several tissues [20]. They also suggested that the prominent observed thyroid signals may be due to Iodine-labeling of LDL. We decided to characterize tissue LDL biodistribution in LDLR-deficient mice treated with the Hd-AdTTR-LDLR-TF vector and therefore expressing the LDLR-TF transgene; we also treated a control group of LDLR-deficient mice with PBS as control. Two weeks after treatment, we administered VivoTag-S 750-labeled LDL nanoparticles to both groups of mice and evaluated fluorescent signals. In mice treated with the LDLR-TF-expressing vector, no signal was present at time 0 (Fig. 6a); 2 hours after fluorescent LDL

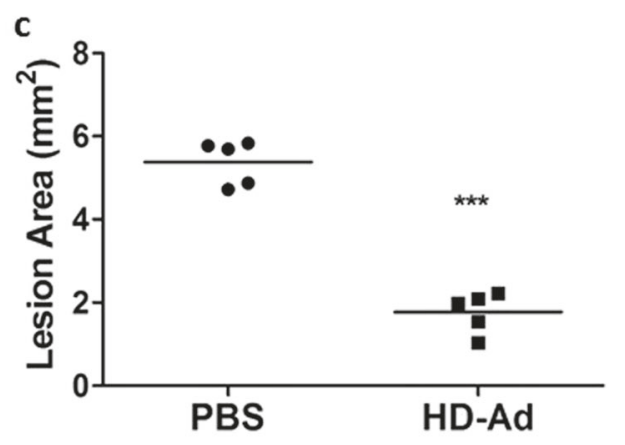

examination, we observed that aortic atherosclerosis lesion areas were smaller in mice treated with HD-Ad/TTR-LDLR-TF (b) compared to the control group (a). Total aortic atherosclerotic lesions were determined and compared in mice from both groups; HD-Ad/TTR-LDLRTF-treated mice (solid square) showed a significantly smaller total atherosclerotic lesion area compared with control mice (solid circle, $* * * p<0.01)$

administration we observed signals in heart, liver, and intestine (Fig. 6b). After 6 hours, the fluorescent signal was still present in the heart, albeit with a lower intensity; a signal appeared in the kidney probably due to the excretion of free dye (Fig. 6c). Signals disappeared $24 \mathrm{~h}$ after fluorescent LDL administration (Fig. 6d). In the control group of mice, we observed a single fluorescence signal in the bladder, probably due to free dye excretion (data not shown).

\section{Discussion}

Over the last two decades, several liver-directed gene therapy strategies have been proposed for LDLR deficiency treatment [13-16]. After unsuccessful attempts with ex vivo gene transfer approaches [30,31], several in vivo gene transfer strategies showed improved efficacy allowing the transduction of a large number of hepatocytes [32]. HD-Ad vector-mediated gene transfer was also successful for this purpose in mice models, showing improved safety and efficacy compared to FG-Ad vectors [33]. After administration of HD-Ad vectors, we and others observed 


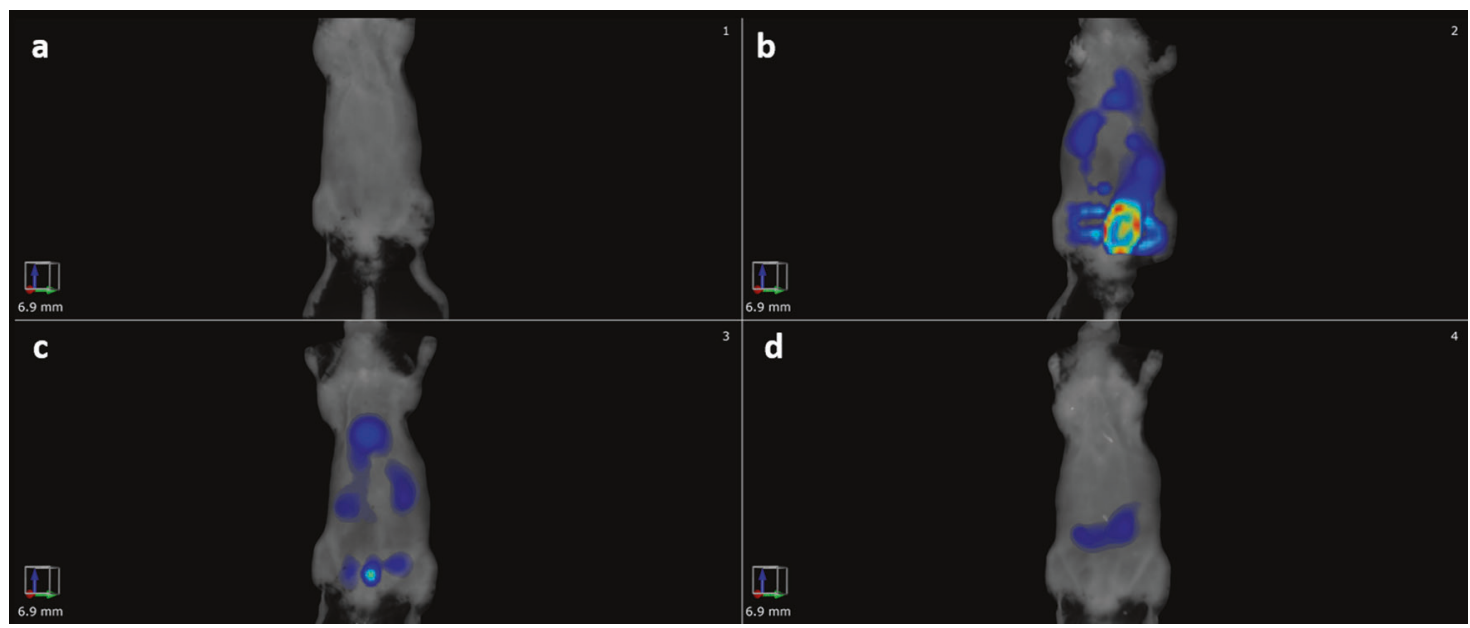

Fig. 6 Low-density lipoprotein (LDL) biodistribution in vivo after HD-Ad-mediated LDLR-TF expression. Fluorescence images in coronal view of LDL receptor (LDLR)-deficient mice treated with $1 \times$ $10 \mathrm{E} 13 \mathrm{vp} / \mathrm{kg}$ of HD-Ad/TTR-LDLR-TF at time $0(\mathbf{a}) ; 2 \mathrm{~h}$ after labeled-
LDL intravenous injection we observed a FMT signal in liver, heart, and intestine (b); $6 \mathrm{~h}$ after injection, we observed a low FMT signal in heart and kidney (c); $24 \mathrm{~h}$ after injection, only a signal in the bladder was present, probably due to free dye excretion (d) extremely prolonged high levels of transgene expression, obtaining life-long disease correction in a number of animal models [13-15]. We have already successfully ameliorated lipid profile and reduced aortic atherosclerosis expressing VLDL receptor in LDLR-deficient mice [15] and hApoA-I in both LDLR- and ApoE-deficient mice [13]. More recently, Greig et al. demonstrated phenotype correction in FH mouse and rhesus monkey models using an AAV8based vector $[17,18]$.

AAV8-derived vectors demonstrated an improved hepatocyte transduction efficiency compared to other AAV serotypes; however, levels of transduction that may lead to clinical improvement are obtained only with administration of very high doses that can cause systemic toxicity [34]. Recently Hordeaux et al. reported the potential for serious acute toxicity in non-human primates after systemic administration of high dose of AAV characterized by thrombocytopenia, hyperbilirubinemia, and increased transaminases [35]. On the other hand, delivery to alternative tissues, such as muscle, may increase the therapeutic window and may lead to safer clinical applications as observed for Glybera, approved for familial hypertriglyceridemia [19]. In order to develop a system that may ultimately be administered in muscle or other tissues, we decided to evaluate an alternative strategy for the correction of LDLR deficiency using a chimeric secreted protein previously engineered by Parise et al. [20]. The LDLR-TF fusion protein contains the signal peptide, the LDL-binding domain, the EGF homology domain, and the O-linked sugar domain of the human LDLR fused with the coding sequence of a rabbit transferrin dimer [21]. This protein induces endocytosis of LDL, through binding of the LDLR$\mathrm{TF}$ fusion protein to the, in fibroblasts obtained from the
LDLR-deficient Watanabe rabbits [21]. Expression of LDLR-TF after hydrodynamic administration of an expression plasmid restored uptake of labeled LDL in liver and other tissues in LDLR-deficient mice [20]. However, the low plasma levels of LDLR-TF obtained after hydrodynamic plasmid administration did not affect neither lipid profile nor atherosclerosis development. This is expected since alteration of lipid metabolism with gene delivery is significantly hampered by the low bioactivity of apolipoproteins and their receptors; in fact, several molecules, such as erythropoietin, induce phenotypic effects at a picograms/ milliliter serum levels range, whereas gene transfermediated expression of apolipoproteins affects lipid profile only when their serum concentration is at least $0.1-1$ $\mathrm{mg} / \mathrm{ml}$. Therefore, gene transfer-mediated LDLR expression for phenotype correction in LDLR mice is effective only when a large percentage of the hepatocytes are corrected [36]; in fact, ex vivo retrovirus-mediated hepatocyte correction and infusion did not lead to clinical improvement in patients for the low number of transduced hepatocytes able to engraft the liver [37, 38].

In the present work, we expressed the LDLR-TF fusion protein using a HD-Ad vector to efficiently transduce a large number of hepatocytes and, therefore, reach significant plasma levels able to affect lipid profile and atherosclerotic lesions in treated LDLR-deficient mice. We generated a cassette for the expression of the LDLR-TF fusion protein under the control of the mouse TTR promoter to increase hepatocyte-specific transcription [39] and subsequently developed the HD-AdTTR-LDLR-TF vector; hepatocyte-specific transduction has been shown to increase transgene duration of expression probably reducing host response against the transgene [40,41]. 
HD-AdTTR-LDLR-TF allowed expression of a functional protein with perinuclear localization suggesting the implication in the secretory pathway. Expression of LDLRTF restored DiI-LDL uptake in CHOldlA7 cells complementing LDLR deficiency. This in vitro preliminary evaluation demonstrated that the fusion protein is able to functionally bind LDL and that the HD-AdTTR-LDLR-TF vector allows the expression of a functional LDLR-TF fusion protein. We then evaluated the effect of HD-AdLDLR-TF administration on the lipid profile in LDLRdeficient mice. Although a lowering of the HDL was also evident, there was a considerable reduction in the TG, TC, and LDL-C levels, suggesting that the HD-AdTTR-LDLRTF treatment led to a marked reduction in the atherogenic potential of the originally highly atherogenic plasma lipoproteins in these animals. These results demonstrate that the ability of the HD-Ad-mediated expression of the LDLR-TF fusion in inducing a long-term modifications of lipid metabolism. Moreover, 12 weeks after treatment, we observed in HD-AdTTR-LDLR-TF vector-treated mice significantly reduced atherosclerotic lesion areas compared to the control group. Since TfRs are expressed in several tissues, this result was not obvious.

Finally, we evaluated biodistribution of fluorescently labeled LDL in vivo after HD-AdTTR-LDLR-TF vector administration and subsequent LDLR-TF chimeric protein expression in LDLR-deficient mice. A similar study had been previously performed by Razzini et al. [20] using iodine-labeled LDL: in this experiment, radioactivity was predominantly taken up by liver and bladder; a significant signal was also observed in the thyroid, probably due to free radioactive iodine uptake that was completely absent in our study probably why our in vivo fluorescence determination, is adequately sensitive and avoid artifacts due to the accumulation of free radioactive iodine in specific organs, such as thyroid. We observed strong signals in liver, but also in heart, intestine, and kidney. TfR 1 is expressed in a number of tissues and organs with a profile similar to LDLR; TfR2, on the other hand, has a liver-restricted expression. At terminal sacrifice, we did not observe significant organ fat accumulation in vector-treated mice after gross pathology examination. However, extra-hepatic uptake of LDL represents a concern for our approach and possible toxicity due to lipid accumulation in other tissues and organs will be thoroughly evaluated in future studies.

The data generated in this work demonstrated that the HD-AdTTR-LDLR-TF vector express a functional LDLRTF fusion protein that is able to bind LDL and induce their uptake both in LDLR-deficient cell line and in LDLRdeficient mice; in this animal model, we observed amelioration of lipid profile and more importantly, reduction of aortic atherosclerosis lesions. To potentiate receptor recycling we are addressing our future studies to block
PSCK9 binding with the extracellular portion our chimeric protein [42].

On the other hand, in order to further improve persistence of transgene expression and reduce toxicity, additional strategies, such as vector PEGylation [43, 44] needs to be explored before suggesting a possible clinical application. However, the major advantage of this strategy is due to the expression of a secreted therapeutic transgene; LDLR-TF chimeric protein can, in fact, be produced by non-hepatic tissues, such as muscle, that can be easily transduced by HD-Ad vectors [45]. The IM delivery route can dramatically improve the safety profile and increase the therapeutic window leading to a safer clinical application.

Acknowledgements The authors wish to acknowledge the memory of Prof. Stefano Ferrari, who has started this project.

\section{Compliance with ethical standards}

Conflict of interest The authors declare that they have no conflict of interest.

Publisher's note: Springer Nature remains neutral with regard to jurisdictional claims in published maps and institutional affiliations.

\section{References}

1. Teramoto T, Shirakawa M, Kikuchi M, Nakagomi M, Tamura S, Surks HK, et al. Efficacy and safety of the cholesteryl ester transfer protein inhibitor anacetrapib in Japanese patients with dyslipidemia. Atherosclerosis. 2013;230:52-60.

2. Vallejo-Vaz AJ, Kondapally Seshasai SR, Cole D, Hovingh GK, Kastelein JJ, et al. Familial hypercholesterolaemia: a global call to arms. Atherosclerosis. 2015;243:257-9.

3. Sniderman AD, Tsimikas S, Fazio S. The severe hypercholesterolemia phenotype: clinical diagnosis, management, and emerging therapies. J Am Coll Cardiol. 2014;63:1935-47.

4. Thomas GS, Cromwell WC, Ali S, Flaim JD, Davidson M. Mipomersen, an apolipoprotein B synthesis inhibitor, reduces atherogenic lipoproteins in patients with severe hypercholesterolemia at high cardiovascular risk: a randomized, double-blind, placebo-controlled trial. J Am Coll Cardiol. 2013;62:2178-84.

5. Tada H, Kawashiri MA, Yoshida T, Teramoto R, Nohara A, Konno $\mathrm{T}$, et al. Lipoprotein(a) in familial hypercholesterolemia with proprotein convertase subtilisin/kexin type 9 (PCSK9) gainof-function mutations. Circ J. 2016;80:512-8.

6. Cupido AJ, Reeskamp LF, Kastelein JJP. Novel lipid modifying drugs to lower LDL cholesterol. Curr Opin Lipidol. 2017; 28:367-73

7. Goulooze SC, Cohen AF, Rissmann R. Lomitapide. Br J Clin Pharmacol. 2015;80:179-81.

8. Stoekenbroek R, Kastelein J, Huijgen R. PCSK9 inhibition: the way forward in the treatment of dyslipidemia. BMC Med. 2015; $13: 258$

9. Cavazzana-Calvo M, Payen E, Negre O, Wang G, Hehir K, Fusil $\mathrm{F}$, et al. Transfusion independence and HMGA2 activation after gene therapy of human $\beta$-thalassaemia. Nature. 2010; 467:318-22.

10. Nathwani AC, Reiss UM, Tuddenham EG, Rosales C, Chowdary P, McIntosh J, et al. Long-term safety and efficacy of factor IX gene therapy in hemophilia B. N Engl J Med. 2014;371:1994-2004. 
11. Brentjens RJ, Davila ML, Riviere I, Park J, Wang X, Cowell LG, et al. CD19-targeted $\mathrm{T}$ cells rapidly induce molecular remissions in adults with chemotherapy-refractory acute lymphoblastic leukemia. Sci Transl Med. 2013;5:177ra38.

12. Jacobson SG, Cideciyan AV, Roman AJ, Sumaroka A, Schwartz $\mathrm{SB}, \mathrm{Heon} \mathrm{E}$, et al. Improvement and decline in vision with gene therapy in childhood blindness. N Engl J Med. 2015;372:1920-6.

13. Oka K, Pastore L, Kim IH, Merched A, Nomura S, Lee HJ, et al. Long-term stable correction of low-density lipoprotein receptordeficient mice with a helper dependent adenoviral vector expressing the very low-density lipoprotein receptor. Circulation. 2001;103:1274-81.

14. Oka K, Mullins C, Kushwaha R, Leen AM, Chan L. Gene therapy for rhesus monkeys heterozygous for LDL receptor deficiency by balloon-catheter hepatic delivery of helper-dependent adenoviral vector. Gene Ther. 2015;22:87-95.

15. Pastore L, Belalcazar LM, Oka K, Cela R, Lee B, Chan L, et al. Helper-dependent adenoviral vector-mediated long-term expression of human apolipoprotein A-I reduces atherosclerosis in apo E-deficient mice. Gene. 2004;327:153-60.

16. Leggiero E, Astone D, Cerullo V, Lombardo B, Mazzaccara C, Labruna $\mathrm{G}$, et al. PEGylated helper-dependent adenoviral vector expressing human Apo A-I for gene therapy in LDLR-deficient mice. Gene Ther. 2013;20:1124-30.

17. Greig JA, Limberis MP, Bell P, Chen SJ, Calcedo R, Rader DJ, et al. Non-clinical study examining AAV8.TBG.hLDLR vectorassociated toxicity in chow-fed wild-type and LDLR $+/-$ rhesus macaques. Hum Gene Ther Clin Dev. 2017;28:39-50.

18. Greig JA, Limberis MP, Bell P, Chen SJ, Calcedo R, Rader DJ. et al. Nonclinical pharmacology/toxicology study of AAV8.TBG. mLDLR and AAV8.TBG.hLDLR in a mouse model of homozygous familial hypercholesterolemia. Hum Gene Ther Clin Dev. 2017;28:28-38.

19. Ferreira V, Petry H, Salmon F. Immune responses to AAV-vectors, the Glybera example from bench to bedside. Front Immunol. 2014;5:82.

20. Parise F, Simone L, Croce MA, Ghisellini M, Battini R, Borghi S, et al. Construction and in vitro functional evaluation of a lowdensity lipoprotein receptor/transferrin fusion protein as a therapeutic tool for familial hypercholesterolemia. Hum Gene Ther. 1999;10:1219-28.

21. Razzini G, Parise F, Calebiro D, Battini R, Bagni B, Corazzari T, et al. Low-density lipoprotein (LDL) receptor/transferrin fusion protein: in vivo production and functional evaluation as a potential therapeutic tool for lowering plasma LDL cholesterol. Hum Gene Ther. 2004;15:533-41.

22. Hymel D, Peterson BR. Synthetic cell surface receptors for delivery of therapeutics and probes. Adv Drug Deliv Rev. 2012;64:797-810.

23. Zhou H, Pastore L, Beaudet AL. Helper-dependent adenoviral vectors. Methods Enxzymol. 2002;346:177-98.

24. Palmer DJ, Ng P. Rescue amplification and large scale production of helper- dependent adenoviral vectors. Cold Spring Harb Protoc. 2011;2011:857-66.

25. Zhou H, Zhao T, Pastore L, Nageh M, Zheng W, Rao XM, et al. A Cre-expressing cell line and an E1/E2a double-deleted virus for preparation of helper-dependent adenovirus vector. Mol Ther. 2001;3:613-22.

26. Toietta G, Pastore L, Cerullo V, Finegold M, Beaudet AL, Lee B. Generation of helper-dependent adenoviral vectors by homologous recombination. Mol Ther. 2002;5:204-10.

27. Krieger M, Brown MS, Goldstein JL. Isolation of Chinese hamster cell mutants defective in the receptor-mediated endocytosis of low density lipoprotein. J Mol Biol. 1981;150:167-84.

28. Cerreto M, Mehdawy B, Ombrone D, Nisticò R, Ruoppolo M, Usiello A, et al. Reversal of metabolic and neurological symptoms of phenylketonuric mice treated with a PAH containing helperdependent adenoviral vector. Curr Gene Ther. 2012;12:48-56.

29. Belalcazar LM, Merched A, Carr B, Oka K, Chen KH, Pastore L, et al. Long-term stable expression of human apolipoprotein A-I mediated by helper-dependent adenovirus gene transfer inhibits atherosclerosis progression and remodels atherosclerotic plaques in a mouse model of familial hypercholesterolemia. Circulation. 2003;107:2726-32.

30. Wilson JM, Chowdhury NR, Grossman M, Wajsman R, Epstein A, Mulligan RC, et al. Temporary amelioration of hyperlipidemia in low density lipoprotein receptor deficient rabbits transplanted with genetically modified hepatocytes. Proc Natl Acad Sci USA. 1990;87:8437-41.

31. Wilon JM, Grossman M, Raper SE, Baker JR Jr, Newton RS, Thoene JG. Ex vivo gene therapy of familial hypercholesterolemia. Hum Gene Ther. 1992;3:179-222.

32. Al-Allaf FA, Coutelle C, Waddington SN, David AL, Harbottle R, Themis M. LDLR-Gene therapy for familial hypercholesterolaemia: problems, progress, and perspectives. Int Arch Med. 2010;3:36.

33. Pastore L, Morral N, Zhou H, Garcia R, Parks RJ, Kochanek S, et al. Use of a liver-specific promoter reduces immune response to the transgene in adenoviral vectors. Hum Gene Ther. 1999;10:1773-81.

34. Vandamme Céline, Adjali Oumeya, Mingozzi Federico. Unraveling the complex story of immune responses to AAV vectors trial after TrialHum. Gene Ther. 2017;28:1061-74.

35. Hordeaux J, Wang Q, Katz N, Buza EL, Bell P, Wilson JM. The neurotropic properties of AAV-PHP.B are limited to C57BL/6J mice. Mol Ther. 2018;7;26:664-8.

36. Piccolo P, Brunetti-Pierri N. Gene therapy for inherited diseases of liver metabolism. Hum Gene Ther. 2015;26:186-92.

37. Grossman M, Raper SE, Kozarsky K, Stein EA, Engelhardt JF, Muller D, et al. Successful ex vivo gene therapy directed to liver in a patient with familial hypercholesterolaemia. Nat Genet. 1994;6:335-41.

38. Faisal AAl-Allaf, Coutelle C, Waddington SN, David AL, Harbottle R, Themis M. LDLR-Gene therapy for familial hypercholesterolaemia: problems, progress, and perspectives. Int Arch Med. 2010;13:3-36.

39. Chuah MK, Petrus I, De Bleser P, Le Guiner C, Gernoux G, Adjali $\mathrm{O}$, et al. Liver-specific transcriptional modules identified by genome-wide in silico analysis enable efficient gene therapy in mice and non-human primates. Mol Ther. 2014;22:1605-13.

40. Castello R, Borzone R, D'Aria S, Annunziata P, Piccolo P, Brunetti-Pierri N, et al. Helper-dependent adenoviral vectors for liver-directed gene therapy of primary hyperoxaluria type 1 . Gene Ther. 2016;23:129-34.

41. Zou L, Zhou H, Pastore L, Yang K. Prolonged transgene expression mediated by a helper-dependent adenoviral vector (hdAd) in the central nervous system. Mol Ther. 2000;2:105-13.

42. Ogura M. PCSK9 inhibition in the management of familial hypercholesterolemia. J Cardiol. 2018;71:1-7.

43. Croyle MA, Le HT, Linse KD, Cerullo V, Toietta G, Beaudet A, et al. PEGylated helper-dependent adenoviral vectors: highly efficient vectors with an enhanced safety profile. Gene Ther. 2005;12:579-87.

44. Wonganan P, Clemens CC, Brasky K, Pastore L, Croyle MA, et al. Species differences in the pharmacology and toxicology of PEGylated helper-dependent adenovirus. Mol Pharm. 2011;8:878-92.

45. Maione D, Della Rocca C, Giannetti P, D'Arrigo R, Liberatoscioli L, Franlin LL, et al. An improved helper-dependent adenoviral vector allows persistent gene expression after intramuscular delivery and overcomes preexisting immunity to adenovirus. Proc Natl Acad Sci USA. 2001;98:5986-91. 